\title{
An Extended Roofline Model with Communication-Awareness for Distributed-Memory HPC Systems
}

\author{
David Cardwell \\ Indiana University-Purdue University Indianapolis \\ dwcardwe@iupui.edu
}

\author{
Fengguang Song \\ Indiana University-Purdue University Indianapolis \\ fgsong@iupui.edu
}

\begin{abstract}
Performance modeling of parallel applications on distributed memory systems is a challenging task due to the effects of CPU speed, memory access time, and communication cost. In this paper, we propose a simple and intuitive graphical model, which extends the widely used Roofline performance model to include the communication cost in addition to the memory access time and the peak CPU performance. This new performance model inherits the simplicity of the original Roofline model and enables performance evaluation on a third dimension of communication performance. Such a model will greatly facilitate and expedite the analysis, development and optimization of parallel programs on high-end computer systems. We empirically validate the extended new Roofline model usingfl oating-point-computation-bound, memory-bound, and communication-bound applications. Three distinct high-end computing platforms have been tested: 1) high performance computing (HPC) systems, 2) high throughput computing systems, and 3) cloud computing systems. Our experimental results with four different parallel applications show that the new model can approximately evaluate the performance of different programs on various distributed-memory systems. Furthermore, the extended new model is able to provide insight into how the problem size can affect the upper bound performance of parallel applications, which is a special property revealed by the new dimension of communication cost analysis.
\end{abstract}

\section{CCS CONCEPTS}

- Theory of computation $\rightarrow$ Distributed computing models; Parallel computing models; $\bullet$ Networks $\rightarrow$ Network performance modeling;

\section{KEYWORDS}

Roofline model, performance prediction, visualization

\section{ACM Reference format:}

David Cardwell and Fengguang Song. 2019. An Extended Roofline Model with Communication-Awareness for Distributed-Memory HPC Systems. In Proceedings of International Conference on High Performance Computing in

Permission to make digital or hard copies of all or part of this work for personal or classroom use is granted without fee provided that copies are not made or distributed for profit or commercial advantage and that copies bear this notice and the full citation on thefi rst page. Copyrights for components of this work owned by others than the author(s) must be honored. Abstracting with credit is permitted. To copy otherwise, or republish, to post on servers or to redistribute to lists, requires prior specific permission and/or a fee. Request permissions from permissions@acm.org.

HPC Asia 2019, January 14-16, 2019, Guangzhou, China

() 2019 Copyright held by the owner/author(s). Publication rights licensed to Association for Computing Machinery.

ACM ISBN 978-1-4503-6632-8/19/01...\$15.00

https://doi.org/10.1145/3293320.3293321
Asia-Pacific Region, Guangzhou, China, fanuary 14-16, 2019 (HPC Asia 2019), 10 pages.

https://doi.org/10.1145/3293320.3293321

\section{INTRODUCTION}

An intuitive performance model that is capable of balancing the major attributes on distributed systems (i.e., communication cost, memory accesses, andfl oating-point computation) would be insightful in evaluating parallel algorithms and new platforms. The original Roofline model [26] provides a simple and intuitive visualization of the relationship between performance and the two attributes of memory accesses andfl oating-point operations.

Despite the Roofline model's wide usage [16] [7] [23] [18] [3], the original model so far does not gauge the effect of the interconnection network on distributed-memory systems. As the communication cost on emerging high-end computing systems becomes more significant, we strive to extend the original Roofline model such that the network bottleneck is considered but without reducing utility with regard to memory-bound or CPU-bound algorithms. We achieve this by defining the concept of communication arithmetic intensity. By doing so, the balance of the three major bottlenecks on a particular high-end system with respect to a specific application can be visualized. The primary benefits of the new model are the simple input parameters and the intuitive visual nature, which are elaborated as follows:

Simple input parameters: The original Roofline model has few input variables and its system-specific parameters are also easy to measure. Similarly, in our new model, we keep it simple and do not require measurements of message send overhead, message receive overhead, message size, or network latency. Instead, we use a ping-pong benchmark to determine the maximum point-to-point bandwidth [15]. This is the only parameter added in addition to the maximumfl oating-point performance and memory bandwidth carried over from the original Roofline model.

A visual model: The original Roofline model is an intuitive visualization. We try to carry this over to the new model, resulting in both a $2 \mathrm{D}$ version and a $3 \mathrm{D}$ visualization of the $2 \mathrm{D}$ model. The $2 \mathrm{D}$ visualization consists of two separate plots that show the network and memory bottlenecks separately. This way both bounds can be visualized independently.

The "Ridge point" is an essential visual part of the original Roofline model and our extended new model, as can be seen in Fig. 1. It is the minimum point where the operational intensity is sufficient to reach the maximumfl oating-point performance. Algorithms with operational intensities to the left of the ridge point are memory-bound, while those to the right arefl oating-point-bound. With the ridge point, the original model is able to identify bottlenecks and predict the performance upper bound on any architecture 


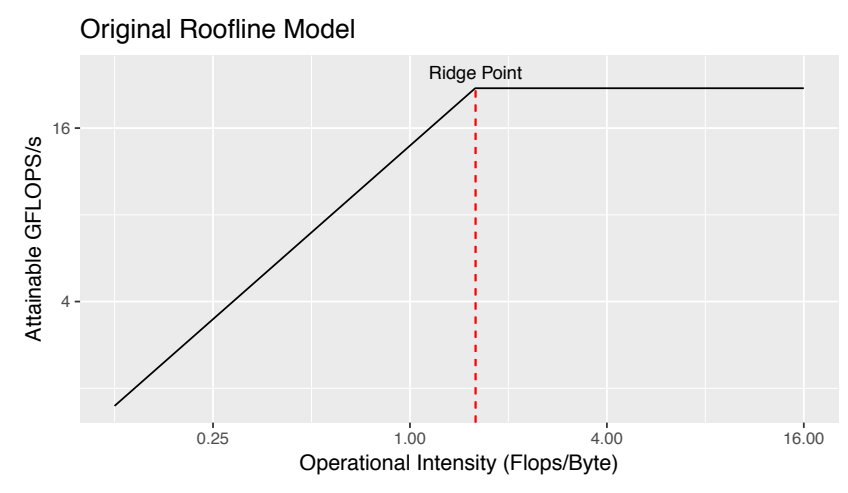

Figure 1: The original Roofline model for Karst, highlighting the ridge point. The ridge point is where the operational intensity is high enough to achieve maximum performance.

without running or profiling the parallel application. We aimed to carry this over to our new model. The new model operates similarly to the original model, where network-bound algorithms have communication arithmetic intensity to the left of the ridge point, while compute-bound or memory-bound problems are to the right, allowing an algorithm to be easily classified.

We validated the new model on four parallel applications (or algorithms) to determine the model's utility. The four applications are: dense matrix-matrix product, 1D fast Fourier transform, 2D 5-point stencil, and Boruvka's minimum spanning tree. These applications represent linear algebra, spectral methods, and structured grids, representing three of the seven motifs of High Performance Computing [2]. The last algorithm represents a classic graph problem.

To verify the model, we tested the performance of the four applications on three high-end computing systems, which represent three different computing paradigms. We selected Big Red II for High Performance Computing, Karst for High Throughput Computing, and Jetstream for Cloud Computing. The specifications of these systems are summarized in Table 4 in Section 4.

To the best of our knowledge, this paper makes the following primary contributions:

- We extended the Roofline model so that it can consider network communication costs and can support distributed memory systems.

- We performed an empirical study and validated the extended new Roofline model on four algorithms of various levels of memory and communication traffic. The results show that our new model provides a tighter upper bound on the attainable performance than the original widely-used Roofline model.

- We explored three different kinds of high-end systems, including HPC, high-throughput, and Cloud computing with respect to distributed algorithms.

\section{BACKGROUND}

The original Roofline model [26] was introduced by Williams and Patterson et al. with the intention of creating a simple and intuitive a) Original Roofline Models

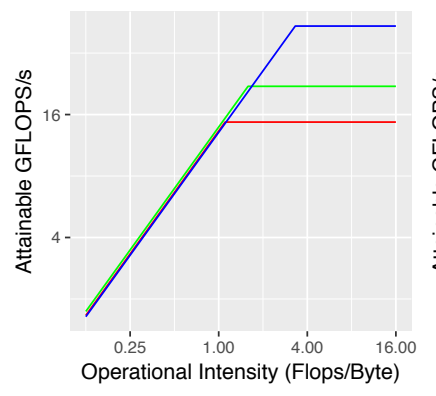

System — Big Red II — Jetstream — Karst b) New Roofline Models

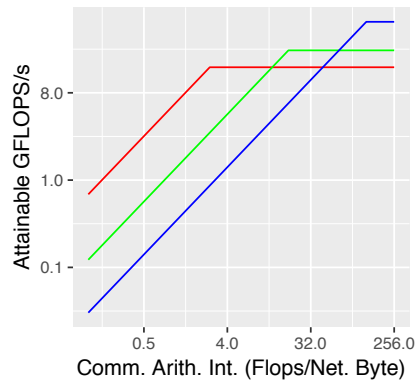

Figure 2: a) Original Roofline models for the three systems, Big Red II, Karst, and Jetstream. b) New Roofline models for the same three systems. Rather than operational intensity on the X-axis we have communication arithmetic intensity.

visual performance model appropriate for shared memory multicore systems. It has been widely adopted by the community due to its capability to make predictions by using such a simple model. It also gives users a simple way of visualizing complex performance issues such as the relationship between memory bandwidth and attainable performance.

The original Roofline model is defined by Equation 1, in which the minimum of the peakfl oating-point performance and the peak memory bandwidth multiplied by the operational intensity determines an upper bound for the attainable performance with respect to GFLOPS/s.

Also, operational intensity is defined as the ratio offl oating-point operations to bytes transferred to and from memory, as defined by Equation 2. Determining the operational intensity of a kernel and inspecting whether it is to the left or right of the ridge point can help users determine the bottleneck that needs to be optimized. For instance, Fig. 2-a shows three examples of the original Roofline model for the three high-end computing systems we test in the paper, respectively. The three colors represent an HPC system, a high-throughput system, and a Cloud system, respectively.

$$
\begin{aligned}
& \text { Attainable GFLOPS } / \mathrm{s}=\operatorname{Min}( \\
& \text { Peak Floating Point Performance, } \\
& \text { Peak Memory Bandwidth } \times \text { Operational Intensity }) \\
& \text { Operational intensity }=\frac{\text { Floating-point operations }}{\text { Memory bytes transferred }}
\end{aligned}
$$

\section{AN EXTENDED NEW ROOFLINE MODEL}

In order to estimate the effects of communication, we build a new communication-aware Roofline model. In the new model, we add a third dimension of communication, and introduce a term called "communication arithmetic intensity" to represent a network equivalent of operational intensity. In this section, wefi rst introduce a $2 \mathrm{D}$ version of the new model, then present a $3 \mathrm{D}$ visualization of it.

\subsection{The New Model}

Equation 3 defines our new Roofline model. 
Attainable GFLOPS/s $=$ Min (

Peak Floating Point Performance,

Peak Communication Bandwidth $\times$ Communication Arithmetic Intensity)

Instead of operational intensity and peak memory bandwidth, we use communication arithmetic intensity and peak communication bandwidth. Communication arithmetic intensity, as seen in Equation 4, equals the number offl oating-point operations per byte of data transferred over the interconnection network.

$$
\text { Communication arithmetic intensity }=\frac{\text { Floating-point operations }}{\text { Network bytes transferred }}
$$

Fig. 2-b displays examples of the new Roofline model for three different distributed-memory systems. In the new Roofline model, if an application has a communication arithmetic intensity to the left of the ridge point, its performance is limited by its communication cost. Also, it implies that users need to decrease the number of messages and number of transferred bytes to reduce the communication cost. Otherwise, it is eitherfl oating-point-bound or memory-bound (for which the original Roofline model may determine whether a kernel is CPU-bound or memory-bound). By examining the ratio of communication tofl oating-point operations we can determine the likelihood that an algorithm is communication-bound. In general, users can use the model tofi nd out what is the most significant performance bottleneck-whether performance is constrained either by memory accesses, by CPU speed, or by network communications.

3.1.1 Simplifications in the Model. Similarly to the original Roofline model, we make several simplifications to make our model straightforward. We consider all communication to be inter-node and synchronous. In this paper, most tested parallel applications use either blocking or collective MPI operations such as MPI_Allgather rather than asynchronous operations such as MPI_Isend. Hence, we consider the most significant type of communication only. Also, like the original Roofline model, we don't model latency for either memory or the interconnection network in order to simplify the model as much as possible.

3.1.2 Differences from the Original Roofline Model. The original Roofline model has more than one bottleneck line (i.e., ceiling) in the plot. This is to consider techniques such as FMA and SIMD. Our model does not add another network Roofline (or ceiling) to the original Roofline plot. Rather, we place the network roofline on a new plot. This is due to differences in the model equations. The $\mathrm{X}$ axis in our new model represents communication arithmetic intensity. This is not analogous to operational intensity, which is the $\mathrm{X}$ axis in the original model. For this reason, we separated the new model from the original model.

In the original model, a parallel application can be either memorybound orfl oating-point bound. In our model, it can also be communication bound. This makes a single 2D Roofline plot unable to differentiate all three bounds. For this reason, we present the new and old models side-by-side.
Table 1: A summary of thefi ve parameters of the model. Derivation refers to whether the parameter is theoretical or taken from benchmark results. Specificity refers to whether the parameter is specific to system hardware or to an application.

\begin{tabular}{||c|c|c||}
\hline Parameter & Derivation & Specificity \\
\hline \hline Peak Floating Point Performance & Benchmark & System-specific \\
\hline Peak Memory Bandwidth & Benchmark & System-specific \\
\hline Peak Communication Bandwidth & Benchmark & System-specific \\
\hline Operational Intensity & Theoretical & Algorithm-specific \\
\hline Communication Arithmetic Intensity & Theoretical & Algorithm-specific \\
\hline
\end{tabular}

\subsection{Specification of the Parameters in the Original and New Models}

The original Roofline model requires three parameters: peakfloatingpoint performance, peak memory bandwidth, and operational intensity. Our new model keeps the peakfl oating-point performance but swaps the other two with peak communication bandwidth and communication arithmetic intensity.

These parameters are derived either from benchmarks or from theory. We use measured benchmark results to determine the three peak related parameters, while using algorithmic analysis to determine operational intensity and communication arithmetic intensity.

On the other hand, the original model uses multi-threaded peak floating-point performance and memory bandwidth, while our model uses single-threaded parameters. We do this to model the performance from the point of view of a single MPI process.

We summarize thefi ve parameters in Table 1 and present how to determine them in the following sections.

\subsection{How to Determine the System-Specific Parameters}

There are three system-specific parameters, as shown in Table 1. Their values can be determined as follows.

Peak Floating-Point Performance: It is determined by calling an optimized level-3 BLAS dgemm function [10].

Peak Memory Performance: The memory bandwidth is determined by running the STREAM benchmark [19]. In the singlethreaded version of the STREAM benchmark, four algorithms are run: copy, scale, sum and triad. We use the highest result from any of the four algorithms.

Peak Network Performance: We wrote a simple ping-pong benchmark to measure the communication bandwidth. In the benchmark, a large message is sent to another compute node, which then responds to the message. We ensured that the processes were allocated to adjacent nodes to minimize the effect of latency. The end-to-end time is then measured using the local timer on thefi rst node. We divide the message size (in bytes) by half of the end-to-end time and determine the bandwidth. The highest performance at any message size is used for the model.

The measured memory bandwidth, network bandwidth and floating-point performance on our three distributed-memory systems are provided in Table 2. These are the values used in this paper for the old and new models. 
Table 2: A summary of the benchmarked system-specific parameters on three different distributed-memory systems.

\begin{tabular}{||c|c|c|c||}
\hline System & Memory (GB/s) & Network (GB/s) & GFLOPS/s \\
\hline \hline Big Red II & 13.4 & 5.7 & 14.7 \\
\hline Karst & 13.9 & 1.2 & 22 \\
\hline Jetstream & 13.1 & 0.34 & 43.4 \\
\hline
\end{tabular}

\subsection{How to Determine the Algorithm-Specific Parameters}

There are two algorithm-related parameters, which require users to conduct specific algorithm analyses. Their values can be decided as follows.

Operational Intensity: Operational intensity is defined as the ratio offl oating-point operations to bytes transferred to and from main memory. To count the number offl oating-point operations, we use widely known asymptotic equations for each algorithm. To count the memory bytes transferred, we use different theoretical methods. In some cases, we use simple input-output byte counting. In other cases, we use more sophisticated methods such as cache-oblivious analysis [21]. We tried to derive realistic counts because our goal is to achieve the best estimation possible.

Communication Arithmetic Intensity: Communication arithmetic intensity is defined as the number offl oating-point operations per byte transferred over the network. For the bytes transferred over the network, we handle point-to-point and collective communications differently to account for the single-process viewpoint of the model.

For point-to-point communications, we sum up the size of the messages. For example, if one process sends a message of size $s$ bytes to a second process, and the second process responds to thefirst with a message of the same size, then the number of bytes is $2 s$. If the number of messages being sent is asymmetric among processes, we consider the worst case. For collective communications, we consider the number of steps required to complete the communication. For example, if the MPI implementation uses a minimum spanning tree algorithm to perform the communication, then it can be estimated to complete in $\lceil\log P\rceil$ steps [25].

\subsection{A 3D Visualization of the Extended Roofline Model}

So far, we have presented our new model as a $2 \mathrm{D}$ visualization that considers communication andfl oating-point bottlenecks. Next, we will combine the original Roofline model with our new model to produce a $3 \mathrm{D}$ visualization that can show the memory bandwidth bottleneck as well. By doing this, we can consider all three bottlenecks in a single visualization.

We define this combined 3D model by Equation 5. Now, the model hasfi ve parameters in total, which are listed as follows: peak floating-point performance, peak memory bandwidth, peak communication bandwidth, operational intensity, and communication arithmetic intensity.

Examples of the 3D model will be shown later in section 7 .

Attainable GFLOPS $/ \mathrm{s}=\operatorname{Min}($

Peak Floating Point Performance,

Peak Memory Bandwidth $\times$ Operational Intensity,

Peak Communication Bandwidth $\times$ Communication Arithmetic Intensity)

\section{RELATED WORK}

The Roofline performance model has gained a lot of attention since it was introduced in 2009. Thereafter a few new models have been built to extend the Roofline model. However, there is almost no Roofline research that studies the network communication cost for distributed memory systems.

The Boat Hull model by Nugteren et al. extends the Roofline model by utilizing algorithmic skeletons to create algorithm-specific Roofline models for shared memory systems [20]. The Cache-aware Roofline model by Ilic et al. changes the chip-centric viewpoint of the original model to a core-centric one [13]. In this way, the full cache hierarchy is considered. Cho et al. extend the model to consider working set size and apply this to embedded systems. Rather than simply considering the peak-performance of the cache as in the Cache-aware Roofline model, their version considers the working set size [6]. Denoyelle et al. also extend the Cache-Aware Roofline Model to consider NUMA systems. While it considers data locality and one-to-all and all-to-all access patterns and bus congestion, their analysis is limited to a single compute node [9].

Cabezas et al. add architectural details such as latency and outof-order execution to the performance model and estimate performance with a simulator by scheduling instructions and performing cycle-level analysis [5]. Similarly to their model we aim to achieve a tighter bound on the predicted performance by considering more details of a system.

Lorenzo et al. introduce a version of the Roofline model by adding a memory latency dimension [17]. Suetterlein et al. extend the model to consider latency hiding and amortized analysis and apply it to asynchronous many-task runtimes [24]. Their model does consider network throughput on distributed memory systems, however, they focus on event-driven tasks rather than the most widely used MPI programming model.

\section{APPLICATIONS}

In this section, we introduce the four applications that we use to verify our new Roofline model. These are dense matrix-matrix product, 1D fast Fourier transform, 2D 5-point stencil, and Boruvka's minimum spanning tree algorithm. All the applications are written in $\mathrm{C}$ or $\mathrm{C}++$ and parallelized using MPI for distributed memory systems. Thefl oating-point operations, memory bytes transferred and network bytes transferred are well-known results for each algorithm [11] [22] [1] [4]. We summarize the derived values of operational intensity and communication arithmetic intensity for each application in Table 3.

Explanation of the Notation Used: In each algorithm except for minimum spanning tree (MST), $N$ represents the input problem size. For MST, the number of edges $E$ and number of vertices $V$ define the problem size. The variable $P$ represents the number of MPI processes for all algorithms.

We briefly introduce the applications below.

(1) Dense Matrix-Matrix Product: Dense Matrix-Matrix product was selected as afl oating-point-operation-bound algorithm, with $S U M M A$ as the implementation [12]. This algorithm implements $C \leftarrow \alpha A B+\beta C$ where $A, B$, and $C$ are doubleprecision matrices. We assume square matrices of size $N$ and using $P$ processes. The kernel implementation uses the 
Table 3: Formulas for calculating the Operational Intensity and Communication Arithmetic Intensity for the applications in this paper.

\begin{tabular}{||c|c|c|}
\hline Name & Operational Intensity & Communication Arithmetic Intensity \\
\hline \hline SUMMA & $\frac{\left(2 N^{3}+2 N^{2}\right) / P}{\left(B\left(1+\left(N^{2} / B\right)+N^{3} /(B \sqrt{M})\right)\right) / P}$ & $\frac{\left(2 N^{3}+2 N^{2}\right) / P}{2 \sqrt{P}\left(8(N / \sqrt{P})^{2} \log \sqrt{P}\right)}$ \\
\hline FFT & $\frac{(5 N \log (N)) / P}{(48 N) / P}$ & $\frac{5 N \log (N)) / P}{32(N / P) \log (P)}$ \\
\hline Stencil & $\frac{4((N-2) / P)(N-2)}{56((N-2) / P)(N-2)}$ & $\frac{4((N-2) / P)(N-2)}{32 N}$ \\
\hline MST & $\frac{(|E| \log |V|) / P}{(B(\operatorname{Sort}(|E|) \log |V|)) / P}$ & $\frac{(|E| \log |V|) / P}{12|V|(\log |V| / 2) \log P+12|V| \log |V|}$ \\
\hline
\end{tabular}

Table 4: The specifications of the test systems.

\begin{tabular}{||c|c|c|c||}
\hline & Big Red II & Karst & Jetstream \\
\hline \hline System Type & HPC & HTC & Cloud \\
\hline Processor Family & AMD Opteron & Intel Xeon & Intel Xeon \\
\hline Processor Model & 6380 & E5-2650 & E-2680v3 \\
\hline Cores Per Socket & 16 & 8 & 12 \\
\hline Sockets Per Node & 2 & 2 & 2 \\
\hline RAM Per Node & $64 \mathrm{~GB}$ & $32 \mathrm{~GB}$ & $128 \mathrm{~GB}$ \\
\hline Network & Gemini & $10 \mathrm{GbE}$ & $10 \mathrm{GbE}$ \\
\hline OS & Cray Linux & Red Hat 6 & CentOS 7 \\
\hline
\end{tabular}

dgemm function from the BLAS library to perform the local multiplication. In Table 3, $B$ is the number of elements held in a cache line and $M$ is the cache size in terms of elements [11].

(2) $1 D$ Fast Fourier Transform: The 1D fast Fourier transform performs the FFT operation on complex double elements. This is done out-of-place, meaning that the transpose is performed using additional memory space.

(3) 2D 5-Point Stencil: Stencil algorithms need low-latency networks to achieve high performance. We selected this application as one of our four for this reason. A 2D 5-point stencil was selected due to its relatively low operational intensity compared to 7-, 19- or 27-point kernels.

(4) Boruvka's Minimum Spanning Tree Algorithm: Minimum spanning tree is a classic graph algorithm with heavy communication requirements. We employed Boruvka's algorithm [14] to implement it in parallel.

\section{EXPERIMENTAL RESULTS}

We evaluate our new model with three distinct computer systems, which represent three different computing paradigms: 1) High Performance Computing, 2) High Throughput Computing, and 3) Cloud Computing. The three systems are Big Red II (HPC), Karst (HTC) and Jetstream (Cloud). Their system specifications are detailed in Table 4.

\subsection{Experiment Setup}

In this subsection, we describe the number of iterations we run our experiments, problem sizes, and number of processes for each application.

For SUMMA, MST and FFT, we ranfi ve trials and recorded the average time. For Stencil, we timed 1000 iterations of the algorithm.
Table 5: The range of problem sizes tested on each system and application respectively.

\begin{tabular}{||c|c|c|c||}
\hline & Big Red II & Karst & Jetstream \\
\hline \hline SUMMA & \multicolumn{3}{|c||}{$242-61,952$} \\
\hline FFT & $256-536,870,912$ & $256-268,435,456$ & $256-67,108,864$ \\
\hline Stencil & \multicolumn{2}{|c|}{$256-131,072$} & $256-65,536$ \\
\hline MST & \multicolumn{3}{|c||}{$65,536-67,108,864$} \\
\hline
\end{tabular}

All problem sizes were varied by multiples of two and varied depending on the system and algorithm. The tested sizes are listed in Table 5 .

We performed two sets of experiments. One set had one process per node, and the other had multiple processes per node. We did the single-process-per-node experiments since MPI uses shared memory for local communication. This means that the local and remote communication speeds are not the same. Due to this difference, we wanted to see if our model would be able to make a prediction in either scenario. The process allocation varied by application and system.

6.1.1 Single Process Per Node. On Big Red II and Karst, the applications other than SUMMA were tested with 128 nodes. For SUMMA, the simplified implementation requires that the process count be a perfect square. In that case 121 nodes were used. Jetstream was not tested with single-process-per-node allocation. This was due to a resource limit of a maximum of 132 processes total among all virtual machines.

6.1.2 Multiple Processes Per Node. The SUMMA application was executed on Big Red II with 121 nodes and 16 processes per node (i.e. a total number of 1936 processes). On the Karst system, we used 36 nodes with 16 processes per node for a total of 576 SUMMA processes. We don't include the experimental results for the other applications due to the space limitations of this paper.

Unlike Big Red II and Karst, Jetstream has virtualized resources. We used m1.xxlarge instances, which are the largest available virtual machine size. Each m1.xxlarge instance has 44 vCPUs and 120GB of RAM available to the user. Due to limits on resource usage, we used three m1.xxlarge instances to perform the computations. In the case of SUMMA, the 121 processes were distributed with 40 processes on two nodes and 41 on the third node. For the other three applications, the 128 processes were distributed as 43 processes on two nodes and 42 processes on the third node.

\subsection{Quantitative Comparison between the Original and New Models}

To determine the quality of the new model, we mustfi rst evaluate the accuracy of the old and new models with respect to collected application performance data. Then, we must compare the accuracy of the old and new models.

6.2.1 How to Evaluate the Model Accuracy. Mean Absolute Percentage Error (MAPE), defined by Equation 6, provides a way to determine the difference [8]. In the equation, $n$ represents the number of data points, actual represents the actual collected data point and predicted represents the performance predicted by the model. 
With MAPE, a smaller error percentage indicates that the predicted bound is closer to the measured actual performance.

Table 6: A table of the percentage change in MAPE values from the original Roofline model to ours. Higher percentages indicate a larger improvement in prediction accuracy. We calculate the percentage change with the formula $100 \times \frac{\text { new-original }}{\text { original }}$.

\begin{tabular}{lll}
\hline Algorithm & System & Percentage Change \\
\hline SUMMA & Big Red II & 58.6 \\
SUMMA & Karst & 95.1 \\
SUMMA & Jetstream & 99.1 \\
1D FFT & Big Red II & 90 \\
1D FFT & Karst & 98.1 \\
1D FFT & Jetstream & 99.4 \\
Stencil & Big Red II & 33.3 \\
Stencil & Karst & 85.4 \\
Stencil & Jetstream & 96 \\
MST & Big Red II & 100 \\
MST & Karst & 100 \\
MST & Jetstream & 100 \\
\hline
\end{tabular}

a

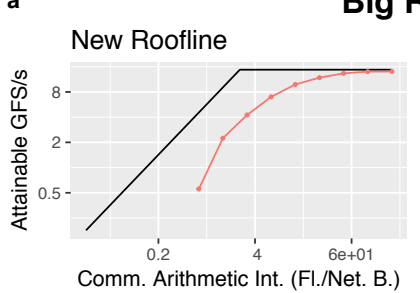

Big Red II

b

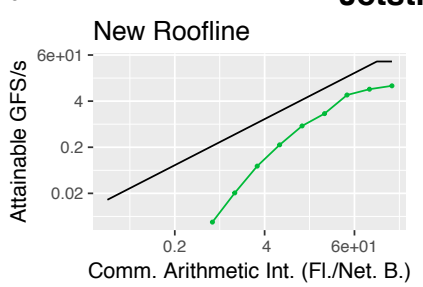

Jetstream

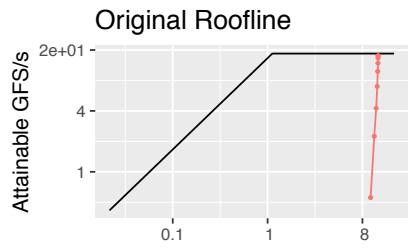

Operational Intensity (Flops/Byte)

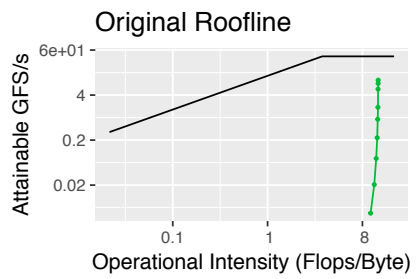

Karst
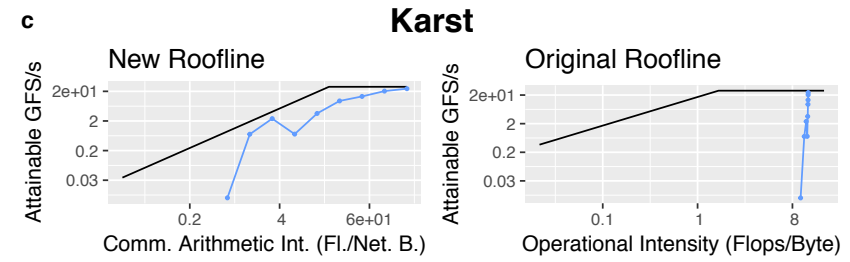

System $\rightarrow$ Big Red II $\rightarrow$ Jetstream $\rightarrow$ Karst

Figure 3: A comparison of the original Roofline model to ours with the SUMMA application. The three systems, Big Red II, Jetstream and Karst are shown in a, b, and c respectively. The black lines represent upper bounds on the attainable performance, while the points represent measured performance. The measured performance points represent the same data and are the identical for both models. Each data point corresponds to a different problem size. It should be noted that the $\mathrm{X}$ - and Y-axis scales are not the same among plots.

$$
M A P E=\frac{1}{n} \sum_{t=1}^{n}\left|\frac{\text { actual }- \text { predicted }}{\text { actual }}\right|
$$

6.2.2 How to Compare the Old and New Models. We can now compare calculated MAPE values for each model. Since the raw MAPE value is not intuitive we use percentage change to compare the change from the old to new models rather than examining the actual MAPE values. Percentage change is defined by the formula $100 \times \frac{\text { new-original }}{\text { original }}$, where new represents the MAPE value for the new model, and original represents the MAPE value for the old model. Due to how the formula works, the best possible value is $100 \%$. A negative percentage would indicate that the new model is worse than the old one.

The error reduction results are summarized in Table 6. The results are listed by the algorithm and system respectively. Stencil on Big Red II improved the least from the old model to the new with a percentage change of $33.3 \%$. MST improved the most with a percentage change of $100 \%$ on all three platforms.

\subsection{Interpretation of the Figures}

We use the plots of SUMMA on Big Red II as an example to explain how to understand the performancefi gures.

The left side of Fig. 3-a is the new Roofline model for Big Red II. The $\mathrm{X}$-axis represents the communication arithmetic intensity, while the Y-axis represents the attainablefl oating-point performance. The black line is the Roofline representing the predicted upper bound on attainable performance with respect to communication arithmetic intensity. The plotted points are measured performance results.

The right side of Fig. 3-a is the original Roofline model. The Xaxis represents the operational intensity, while the Y-axis represents the attainablefl oating-point performance. The black line is the Roofline representing the predicted upper bound on attainable performance with respect to operational intensity.

In addition to thefi gures for the models, we also havefi gures for the percentage of communication time during execution for each application and platform. This is to help determine if the new model is giving a reasonable approximation of the level of communication bottleneck. Fig. 4-a is a plot of the percentage of execution time spent on network communication while executing SUMMA on all three platforms. The X-axis is the problem size, and the Y-axis is the percentage communication time. Big Red II, Jetstream and Karst are represented by red, green and blue, respectively.

\subsection{Evaluation Results on Four Applications}

6.4.1 Dense Matrix-Matrix Product (SUMMA). Its corresponding result is shown in Fig. 3. The original Roofline model on the right side of thefi gure shows an operational intensity that is relatively constant around the value of 8 . The smaller matrix sizes have lower performance, and the measured performance points increase in a nearly straight line towards the Roofline as the input size increases. Since the operational intensity does not change, the big performance change at different matrix sizes cannot be explained by the original Roofline model, which always predicts maximum floating-point performance at any matrix size. The original Roofline 
a) SUMMA

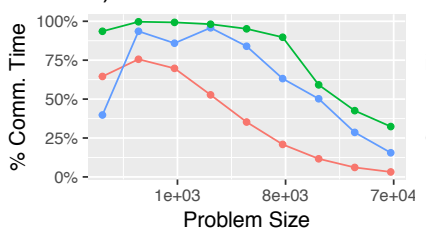

c) Stencil

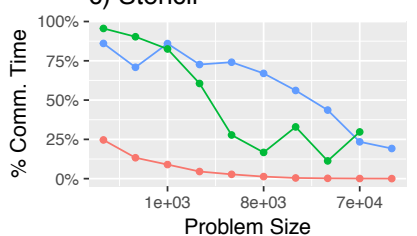

Problem Size

System $\rightarrow$ Big Red II $\rightarrow$ - Jetstream $\rightarrow$ Karst

Figure 4: The percentage of time spent on communication for each algorithm and system. Each subfigure is one application and each color is one system. Big Red II is represented by red, Jetstream is represented by green, and Karst is represented by blue. For Big Red II and Karst, one process per node is used. This gives a total of 121 processes for SUMMA and 128 for the other three applications. 3 nodes were used for SUMMA on Jetstream, with 40 processes on two of the nodes and 41 on the third for a total of 121 . For the other applications on Jetstream 43 processes were used for two of the nodes and 42 on the third for a total of 128 .

model predicts maximum performance since all points are to the right of the ridge point.

On the left side of Fig. 3 we have the new model, where the communication arithmetic intensity varies depending on the matrix size. The points curve from the left side of thefi gures rises up towards the new Roofline as the matrix size increases. This shows that the ratio offl ops to communication bytes is not constant and varies depending on the matrix size. Therefore, communication arithmetic intensity provides an explanation for the low performance at small matrix sizes that the original Roofline model does not provide. More points are located to the left of the ridge point compared to the original Roofline model, giving a better performance analysis.

Also, by looking at Fig. 4-a, we can verify that small matrix sizes are bottlenecked by communication time. On all three platforms, the percentage of communication time is highest at small sizes and decreases to a minority of the total execution time as the problem size increases.

Our model improves over the original Roofline model by $58.6 \%$, 95.1\% and 99.1\% on Big Red II, Karst, and Jetstream respectively, indicating that the new model produces a tighter bound on the attainable performance prediction.

6.4.2 1D Fast Fourier Transform. Fig. 5 shows the results for the 1D fast Fourier transform. Unlike SUMMA, FFT does not have a relatively constant operational intensity. The operational intensity increases as the problem size increases, and the data points pass over the ridge point on Big Red II and Karst, which implies that the application is not memory-bound at large problem sizes.

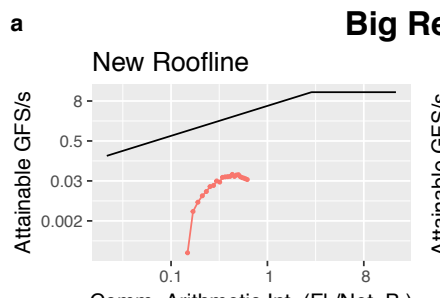

Big Red II
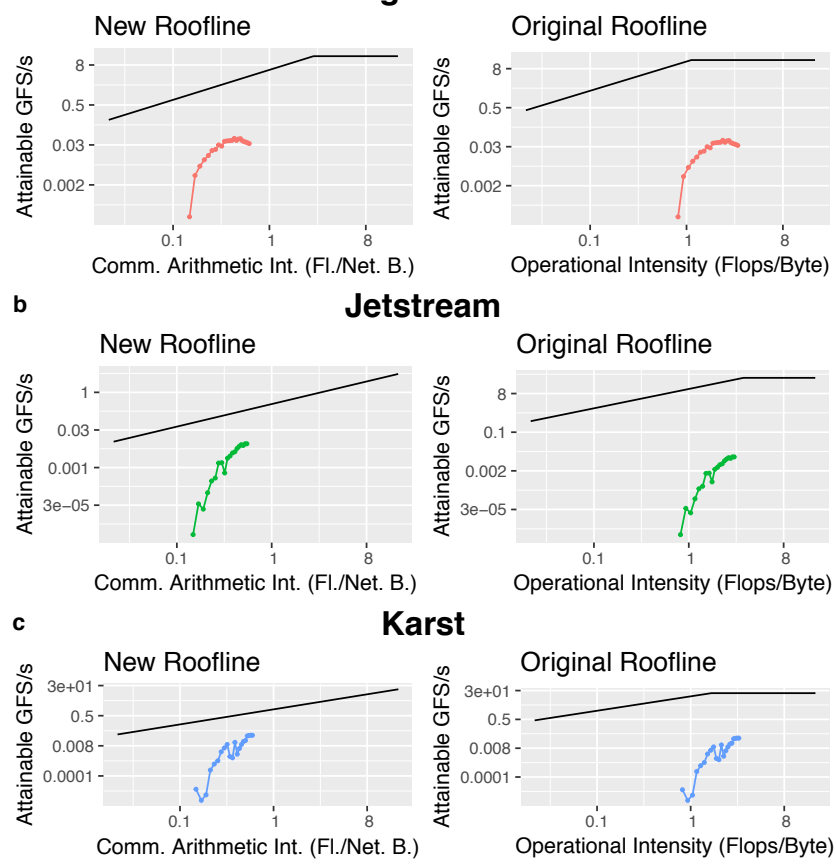

System - Big Red II - Jetstream - Karst

Figure 5: A comparison of the new and original Roofline models for FFT.

On the other hand, the communication arithmetic intensity also increases as the problem size increases. Comparing the right and left results shows that the operational intensity and communication arithmetic intensity values are similar for each data point respectively.

By examining all the left-handfi gures, we canfi nd that the new model gives a lower performance estimate than the original model (see their Y-axises). This occurs because the communication arithmetic intensity value for each point is lower than the equivalent operational intensity value, indicating that the algorithm is more communication-bound than memory-bound. In fact, Fig. 4 has shown that FFT spends the majority of the time on communication at small problem sizes. The percentage of communication time decreases as the problem size increases. This phenomenon agrees with the prediction of our new model.

The percentage change from the old model to the new is $90.0 \%$ on Big Red II, while on Karst and Jetstream the change is $98.1 \%$ and $99.4 \%$, respectively.

6.4.3 2D 5-Point Stencil. The results for Stencil are displayed in Fig. 6. On the right-hand plots, the measured performance points increase quickly upwards since the operational intensity isfi xed around the value of 1 . Hence, the original Roofline model does not give explanations for slower performance at smaller problem sizes.

With our new Roofline model, the communication arithmetic intensity increases as the problem size increases, as shown in the left-hand plots. Fig. 4-c also shows that the percentage of communication time is reduced as the problem size increases, resulting 


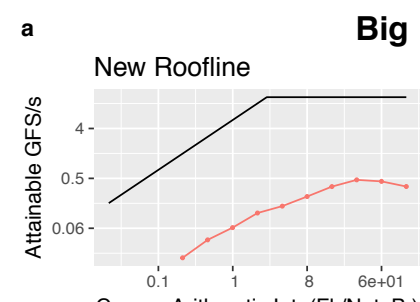

Big Red II

b
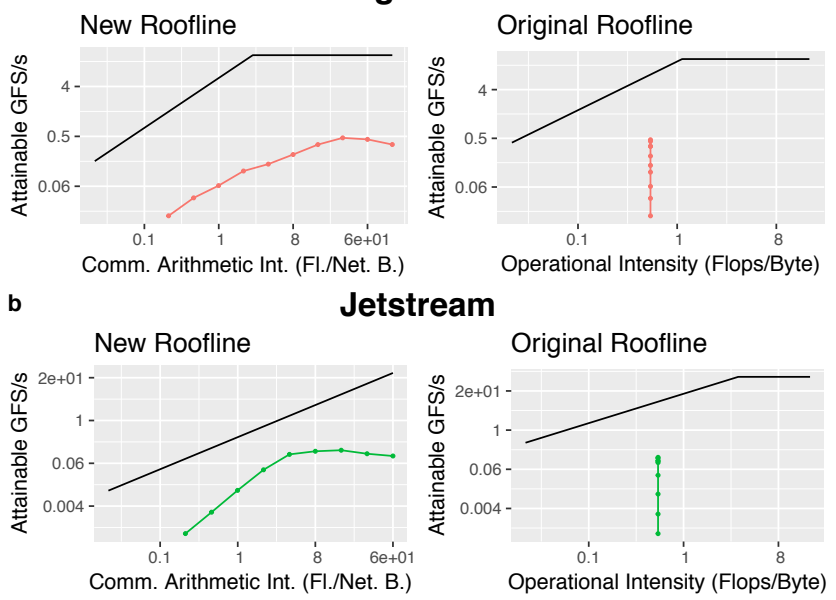

etstream
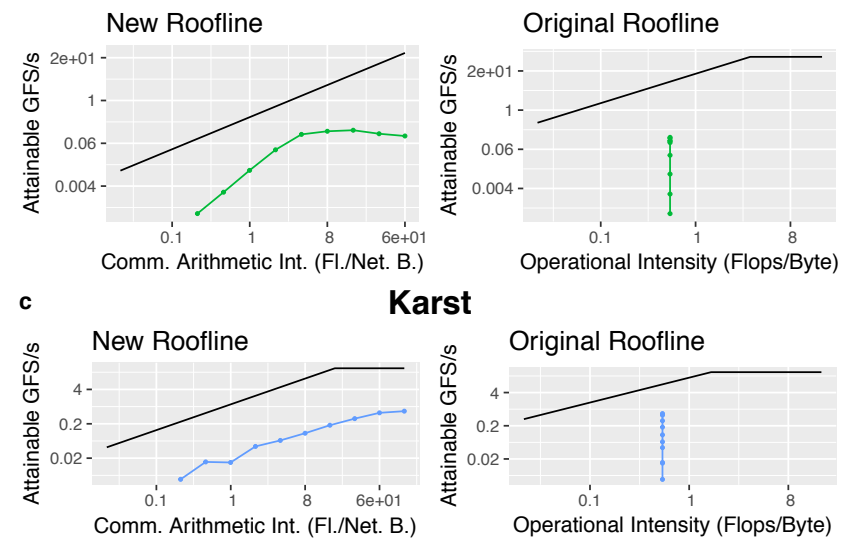

System - Big Red II - Jetstream $\rightarrow$ Karst

Figure 6: A comparison of the new and original Roofline models for Stencil.

in higher communication arithmetic intensities and higher performance. Again, this phenomenon agrees with the prediction of the new model.

The percentage change from the original to the new model is $33.3 \%, 85.4 \%$, and $96.0 \%$ on Big Red II, Karst and Jetstream respectively.

6.4.4 Boruvka's Minimum Spanning Tree (MST). Finally, in Fig. 7 , the operational intensity is much higher than the communication arithmetic intensity, indicating that the algorithm is more communication-bound than memory-bound. From Fig. 4-d, we can see that a relatively constant proportion of the execution time is spent on communication regardless of problem size. This conforms to our new model, where the result points are clustered in a small area. The clustering suggests that the communication arithmetic intensity does not change significantly as the problem size increases for this algorithm.

Our extended new model produces a good prediction for this algorithm with percentage change values of $100 \%$ for all three platforms.

\subsection{Results with Multiple Processes per Node}

The previous experiments use one process per node. In order to further validate the new model, we also test our new model in a multiprocess per node setting.

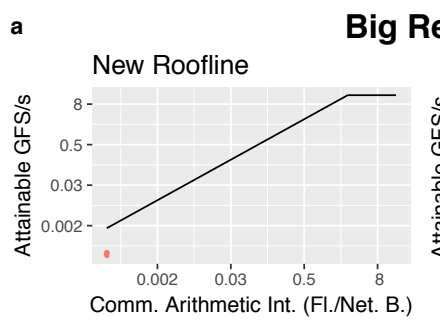

Big Red II
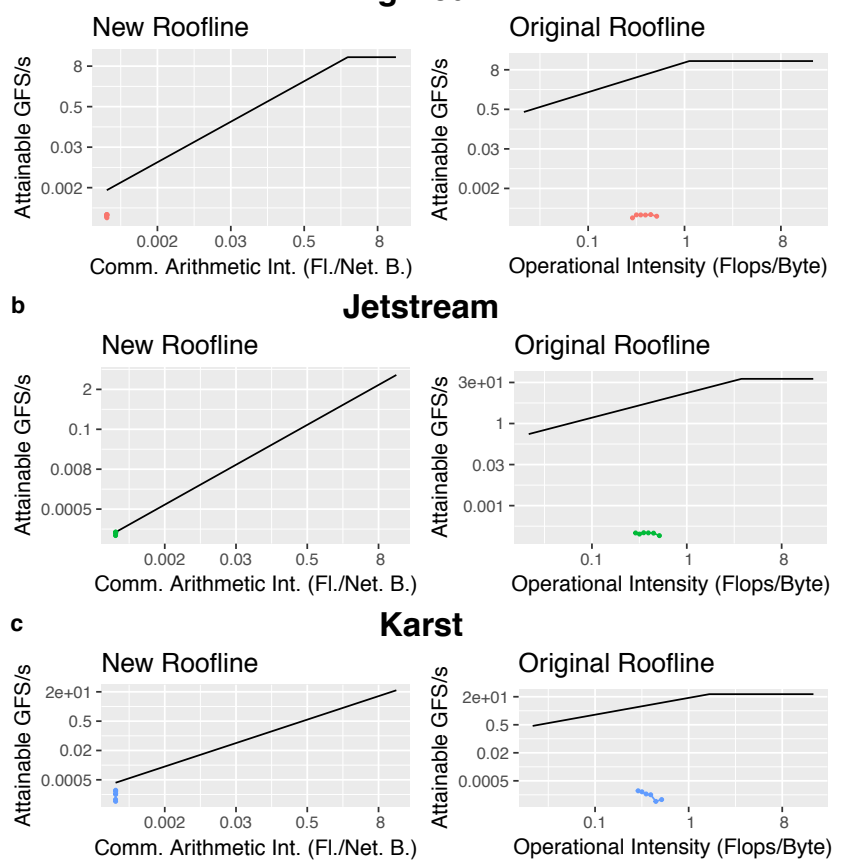

System - Big Red II - Jetstream - Karst

Figure 7: A comparison of the new and original Roofline models for MST.
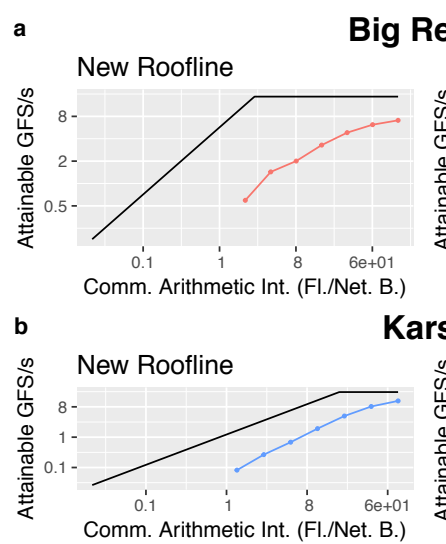

Karst
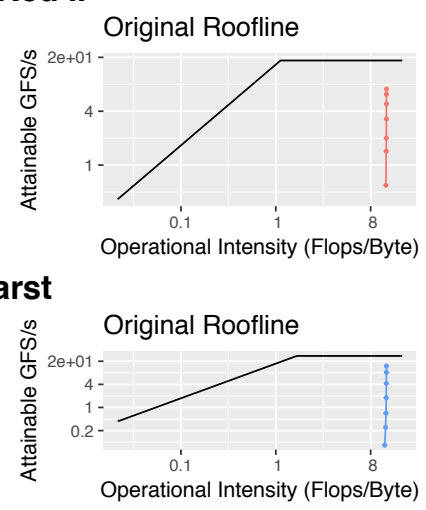

System $\rightarrow$ Big Red II $\rightarrow$ Jetstream $\rightarrow$ Karst

Figure 8: Multi-process per node results for SUMMA.

Since the previous experiments on the Cloud computing Jetstream system already allocated multiple processes per node, Jetstream is not included in this subsection. Also, due to lack of space, we only show the results for SUMMA on Big Red II and Karst as the other applications behave similarly.

Fig. 8 shows the result for SUMMA. The original Roofline model gives the same prediction as in the single-process-per-node results, in which the same maximum performance is predicted at each 
matrix size. By contrast, our new model on the left side significantly improves on this prediction since some of the points have moved to the left of the ridge point for both platforms. Since collective MPI operations block until all processes have synchronized, our model gives a similarly accurate prediction as the single process per node results.

Comparing this result to the single single-process-per-node result from Fig. 3 shows that this result is less communication-bound. This is indicated by the multi-process per node results having consistently higher intensity for each data point respectively. This occurs since although the collective operations are blocking, there is less communication between nodes, which is significantly slower than communication between processes within a node.
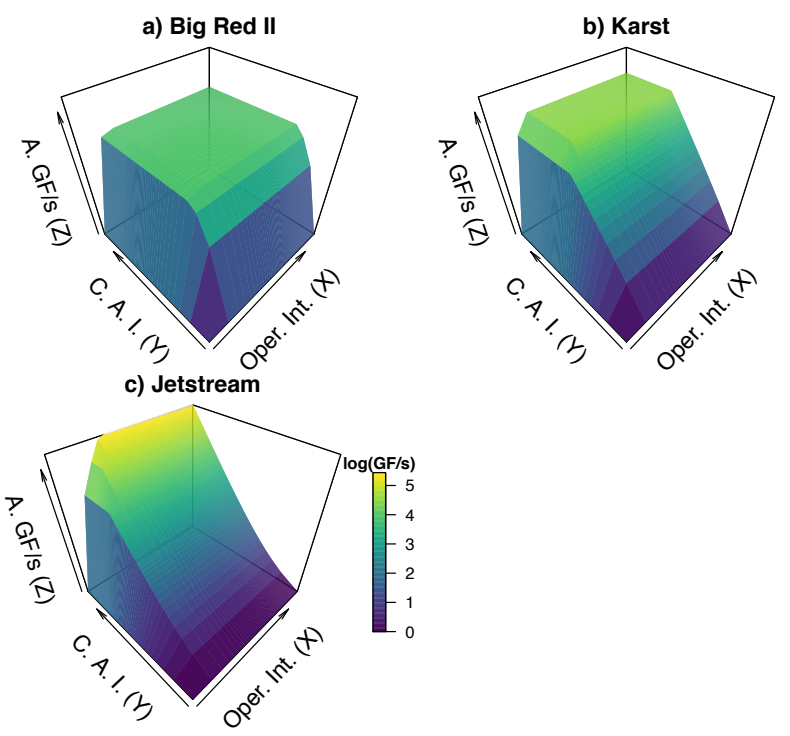

Figure 9: An example of using the 3D model to compare the three systems. C.A.I. refers to communication arithmetic intensity. The $\mathrm{Z}$-axis represents the attainable GFLOPS/s. The models are colored according to the attainable performance at any combination of operational intensity and communication arithmetic intensity.

\section{3D EXPERIMENTAL RESULTS}

Section 6 evaluates the original and new Roofline models as 2D plots. In this section, wefi rst compare the three computer systems (i.e., Big Red II, Karst and Jetstream), then show a new Roofline perspective using the 3D model.

\subsection{Comparison of the Three Computer Systems Using the 3D Model}

Fig. 9 shows the three systems' Roofline models in 3D. In the visualization, the $\mathrm{X}$-axis represents Operational Intensity (i.e., Oper. Int.), the Y-axis represents Communication Arithmetic Intensity (i.e., C.A.I.), and the Z-axis represents the Attainable Floatingpoint performance (i.e., At. GF/s).

In Fig. 9, each computer system'sfi gure consists of three planes: the left, the right, and the top planes.
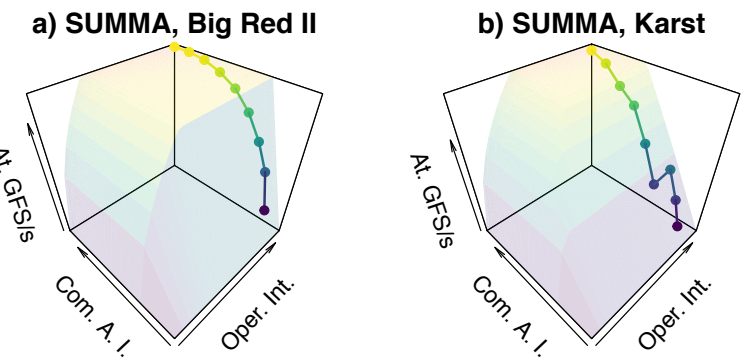

c) SUMMA, Jetstream

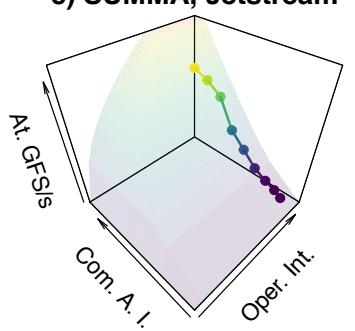

Figure 10: 3D Roofline models for SUMMA on the three target systems. The performance result points are plotted as well as the Roofline surface.

The left plane shows that given afi xed Operational Intensity, the Attainable GF/s will rise as the Communication Arithmetic Intensity increases. On the other hand, the right plane shows that given afi xed Communication Arithmetic Intensity, the Attainable GF/s will change depending on the Operational Intensity. As a result, these three planes combined can produce a 3D volume indicating the maximum performance at any combination of operational and communication arithmetic intensities. The two intensities can also reveal the most significant bottleneck. For instance, an application that has a high operational intensity and a low communication arithmetic intensity is destined to be communication-bound. Therefore, finding ways to reduce communications is more likely to improve the application's performance than tuning memory operations.

If we compare the right planes of the three systems as shown in Fig. 9, we canfi nd that different systems require distinct communication arithmetic intensities (C.A.I.) to attain the maximum floating-point performance. For instance, Jetstream's left plane has the gentlest slope (or the longest path) to reach the peak, Karst has a steeper slope, and Big Red II has the steepest (or shortest) slope. This is because Jetstream has the slowest network, Karst is faster than Jetstream, and Big Red II has the fastest network, as can be seen in Table 2. The implication is that an application on Jetstream must have a very high communication arithmetic intensity to achieve the maximum performance, while Karst and Big Red II have less stringent requirements.

Next, we look at the left planes. Since the memory bandwidths of the three computer systems are similar, all the systems' left planes appear to be the same, as was expected. Finally, Fig. 9 shows that the three systems have various levels of maximumfl oating-point performance. The position of Big Red II's top plane is the lowest. Karst has a top plane with a height in the middle and Jetstream has the highest top plane. 


\subsection{An Example of SUMMA in 3D}

We use the dense matrix-matrix product (SUMMA) application as an example to show how an application's peak performance can be bounded by our new 3D Roofline model. Fig. 10 displays the results of SUMMA on three computer systems. Here, we omit the results of the other applications due to limited space. Each computer system has a 3D subfigure-(a), (b), (c)-which consists of the the models from Fig. 9 together with the measured performances (i.e., the dot points) superimposed. In thesefi gures, the operational intensity and communication arithmetic intensity axes range from 0 to the maximum operational intensity or communication arithmetic intensity value for a given application. Therefore, thesefi gures look dissimilar from those in Fig. 9 since thosefi gures extend to the minimum operational intensity and communication arithmetic intensity required for Jetstream to reach maximum performance. This was done so that the points are easy to interpret rather than being clustered into a small portion of thefi gure.

In addition, the subfigures in Fig. 10 are divided into 64 segments for the purpose of colorization, while the subfigures in Fig. 9 are divided into 128 segments. More segments were used in Fig. 9 to show additional detail that would make the points in Fig. 10 more difficult to see clearly. The measured data points are the same as those used in the 2D Fig. 3. Our objective is to show that the measured data points approximately follow the Roofline surface, with the Roofline surface working as the upper bound. Since the measured data points are bounded by the Roofline in Fig. 3, they are below the Roofline surface as shown in Fig. 10. This is also because the $3 \mathrm{D}$ visualization is essentially a superset of the $2 \mathrm{D}$ plots.

\section{CONCLUSION}

In summary, we have developed an extended new Roofline model to predict parallel program performance on distributed memory systems. In order to achieve the goal, we incorporated the cost of network communication in addition to the cost of main memory accesses. We validated the new model in an empirical study across multiple parallel application classes on three different types of highend computing systems (i.e., high performance, high throughput, and Cloud computing). The extended new Roofline model is kept nearly as simple as the original model so that it can be intuitively visualized in both 2D and 3D diagrams for both algorithm and distributed system performance analyses, providing a convenient way to facilitate understanding the performance of parallel applications especially on distributed-memory systems.

\section{ACKNOWLEDGEMENT}

This work is partially supported by the NSF Award \#1835817, and used the Extreme Science and Engineering Discovery Environment (XSEDE), which is supported by NSF grant number ACI- 1548562.

\section{REFERENCES}

[1] L. Allulli. 2007. Cache Oblivious Computation of Shortest Paths: Theoretical and Practical Issues.

[2] K. Asanovic, J. Wawrzynek, D. Wessel, K. Yelick, R. Bodik, J. Demmel, T. Keaveny, K. Keutzer, J. Kubiatowicz, N. Morgan, D. Patterson, and K. Sen. 2009. A view of the parallel computing landscape. Commun. ACM 52, 10 (Oct. 2009), 56.

[3] A. Bhatelé, L. Wesolowski, E. Bohm, E. Solomonik, and L. V. Kalé. 2010. Understanding Application Performance via Micro-benchmarks on Three Large
Supercomputers: Intrepid, Ranger and Jaguar. The International fournal of High Performance Computing Applications 24, 4 (May 2010), 411-427.

[4] G. S. Brodal. 2004. Cache-Oblivious Algorithms and Data Structures. In Algorithm Theory - SWAT 2004, 9 th Scandinavian Workshop on Algorithm Theory, Humlebaek, Denmark, July 8-10, 2004, Proceedings. 3-13.

[5] V. C. Cabezas and M. Puschel. 2014. Extending the roofline model: Bottleneck analysis with microarchitectural constraints. In 2014 IEEE International Symposium on Workload Characterization (IISWC). IEEE.

[6] S. J. Cho, S. H. Yun, and J. W. Jeon. 2014. A roofline model based on working set size for embedded systems. IEICE Electronics Express 11, 15 (2014).

[7] J. W. Choi, D. Bedard, R. Fowler, and R. Vuduc. 2013. A Roofline Model of Energy. In 2013 IEEE 27th International Symposium on Parallel and Distributed Processing. IEEE.

[8] A. de Myttenaere, B. Golden, B. Le Grand, and F. Rossi. 2016. Mean Absolute Percentage Error for regression models. Neurocomputing 192 (June 2016), 38-48.

[9] N. Denoyelle, B. Goglin, A. Ilic, E. Jeannot, and L. Sousa. 2017. Modeling Large Compute Nodes with Heterogeneous Memories with Cache-Aware Roofline Model. In Lecture Notes in Computer Science. Springer International Publishing, 91-113.

[10] J. J. Dongarra, J. D. Cruz, S. Hammerling, and I. S. Duff. 1990. A Set of Level 3 Basic Linear Algebra Subprograms. ACM Transactions on Mathematical Software (TOMS) 16, 1 (March 1990), 1-17.

[11] M. Frigo, C. E. Leiserson, H. Prokop, and S. Ramachandran. 1999. Cache-Oblivious Algorithms. In Proceedings of the 40th Annual Symposium on Foundations of Computer Science (FOCS '99). IEEE Computer Society, Washington, DC, USA, $285-298$.

[12] R. A. Van De Geijn and J. Watts. 1997. SUMMA: scalable universal matrix multiplication algorithm. Concurrency: Practice and Experience 9, 4 (April 1997), 255-274.

[13] A. Ilic, F. Pratas, and L. Sousa. 2014. Cache-aware Roofline model: Upgrading the loft. IEEE Computer Architecture Letters 13, 1 (Jan. 2014), 21-24.

[14] Paul Jähne. 2015. Erzeugung minimaler Spannbäume auf ungerichteten, kantengewichteten Graphen mit den Algorithmen von Kruskal, Prim und Boruvka. In 45. Jahrestagung der Gesellschaft für Informatik, Informatik 2015, Informatik, Energie und Umwelt, 28. September - 2. Oktober 2015 in Cottbus, Deutschland. 1937-1947.

[15] T. Kielmann, H. E. Bal, and K. Verstoep. 2000. Fast Measurement of LogP Parameters for Message Passing Platforms. In Proceedings of the 15 IPDPS 2000 Workshops on Parallel and Distributed Processing (IPDPS '00). Springer-Verlag, London, UK, UK, 1176-1183.

[16] K. Kim, K. Kim, and Q. Park. 2011. Performance analysis and optimization of three-dimensional FDTD on GPU using roofline model. Computer Physics Communications 182, 6 (June 2011), 1201-1207.

[17] O. G. Lorenzo, T. F. Pena, J. C. Cabaleiro, J. C. Pichel, and F. F. Rivera. 2014. 3DyRM: a dynamic roofline model including memory latency information. The fournal of Supercomputing 70, 2 (March 2014), 696-708.

[18] D. Marques, H. Duarte, A. Ilic, L. Sousa, R. Belenov, P. Thierry, and Z. A. Matveev. 2017. Performance Analysis with Cache-Aware Roofline Model in Intel Advisor. In 2017 International Conference on High Performance Computing \& Simulation (HPCS). IEEE.

[19] J. D. McCalpin. 1995. Memory Bandwidth and Machine Balance in Current High Performance Computers. IEEE Computer Society Technical Committee on Computer Architecture (TCCA) Newsletter (Dec. 1995), 19-25.

[20] C. Nugteren and Henk Corporaal. 2012. The Boat Hull Model: Adapting the Roofline Model to Enable Performance Prediction for Parallel Computing. In Proceedings of the 17th ACM SIGPLAN Symposium on Principles and Practice of Parallel Programming (PPoPP '12). ACM, New York, NY, USA, 291-292.

[21] G. Ofenbeck, R. Steinmann, V. Caparros, D. G. Spampinato, and M. Puschel. 2014. Applying the roofline model. In 2014 IEEE International Symposium on Performance Analysis of Systems and Software (ISPASS). 76-85.

[22] A. Pedram, J. D. Mccalpin, and A. Gerstlauer. 2014. A Highly Efficient Multicore Floating-Point FFT Architecture Based on Hybrid Linear Algebra/FFT Cores. F. Signal Process. Syst. 77, 1-2 (Oct. 2014), 169-190.

[23] D. Rossinelli, C. Conti, and P. Koumoutsakos. 2011. Mesh-particle interpolations on graphics processing units and multicore central processing units. Philosophical Transactions of the Royal Society A: Mathematical, Physical and Engineering Sciences 369, 1944 (May 2011), 2164-2175

[24] J. D. Suetterlein, J. Landwehr, A. Marquez, J. Manzano, and G. R. Gao. 2016. Extending the Roofline Model for Asynchronous Many-Task Runtimes. In 2016 IEEE International Conference on Cluster Computing (CLUSTER). IEEE.

[25] R. Thakur and W. D. Gropp. 2003. Improving the Performance of Collective Operations in MPICH. In Recent Advances in Parallel Virtual Machine and Message Passing Interface. Springer Berlin Heidelberg, 257-267.

[26] S. Williams, A. Waterman, and D. Patterson. 2009. Roofline: an insightful visual performance model for multicore architectures. Commun. ACM 52, 4 (April 2009), 65-76. 